\title{
FEA Practical Illustration of Mesh-Quality-Results Differences between Structured Mesh and Unstructured Mesh
}

\author{
Amir Javidinejad \\ Department of Stress Engineering, Zodiac Aerospace, 7330 Lincoln Way, Garden Grove, CA 92842, USA \\ Correspondence should be addressed to Amir Javidinejad, amirjavidinejad@gmail.com
}

Received 1 May 2012; Accepted 7 June 2012

Academic Editors: J. Clayton and C. Del Vecchio

Copyright () 2012 Amir Javidinejad. This is an open access article distributed under the Creative Commons Attribution License, which permits unrestricted use, distribution, and reproduction in any medium, provided the original work is properly cited.

A reliable design via FEA techniques is highly dependent on the execution of an analysis which is accurate and represents the design precisely. The accuracy and efficiency of the two available methods of meshing of the design components (mapped and free meshing) for finite element analysis are addressed in this paper. This paper intends to clarify that the "shape" of the elements rather than the "pattern" is the distinguishing factor for accuracy of the mesh-quality-results. This paper concludes by comparison of the FEA analysis to the analytical theory that element integrity and results are not governed by the elemental aspect ratio alone. The mesh density has a greater effect. Four types of mesh are investigated and compared for results' accuracy. In specific, a low-density free mesh, a high-density free mesh, a mapped mesh with high aspect ratio, and a mapped mesh with low aspect ratio of a plate with center hole are examined and compared. A plate with center hole is selected for this purpose since its far-field structural behavior is predictable and is highly applicable in showing the differences in accuracy among the different meshing methods.

\section{Introduction}

The ambiguity surrounding the selection of the type of the mesh that would produce highly accurate results has been there for every stress analyst. In this paper, an effort has been made to clear up this ambiguity in a very practical manner via examples and relative comparisons. In general, there are two distinct types of meshing available: (1) free (unstructured) meshing and (2) mapped (structured) meshing. The free meshing produces a "free" type of shape and pattern for element geometry. The free shape and pattern meshing assumes a less accurate result production since the element's behavior is defined to be of a lower quality. The mapped-mesh is distinct in shape by uniformity and consistent in pattern by repeatability. This type of meshing is desirable because it produces a computationally wellbehaved mesh [1].

The free meshing can usually produce high aspect ratio type of mesh, where the ratio of the long side of the elements to the short side is large. This type of element geometry leads to inaccuracy and nonconvergence of the solution [2]. To increase the finite element performance, mapped meshing is utilized to control the aspect ratios of the elements and to produce a consistent mesh. Other advanced techniques such as adaptive meshing [3] are also developed to estimate the errors and eliminate high aspect ratio meshes.

Thus, a lot of emphasis is put on structured mesh generation in finite element analysis. Here, in this paper four different possible meshes consisting of structured and unstructured mesh are examined. The platform in which this investigation is done is a plate with a center-hole. In specific, a 20 inch by 20 inch in square plate with thickness of 0.2 inches is selected. The plate contains a center hole located symmetrically at the center with a radius of 2 inches. Further, an applied tensile loading of $1000 \mathrm{lbf}$ is applied to the plate in the horizontal $x$-direction.

A plate with center hole model is utilized since its stress behavior around the center hole is predictable. There are many experimental investigations performed $[4,5]$ that define and investigate the stress behavior of the plates with centrally located holes. Normally, the stress level perpendicular to the horizontal axis of the plate (perpendicular to the loading direction) is at maximum at exactly 90 degrees angular location to the center-hole circumference. This is where stress concentration is developed. Theoretically, this stress level magnitude is at about three times the stress developed in the plate thickness, due to the tensile loading of the plate. A theoretical analysis follows to develop the closed 


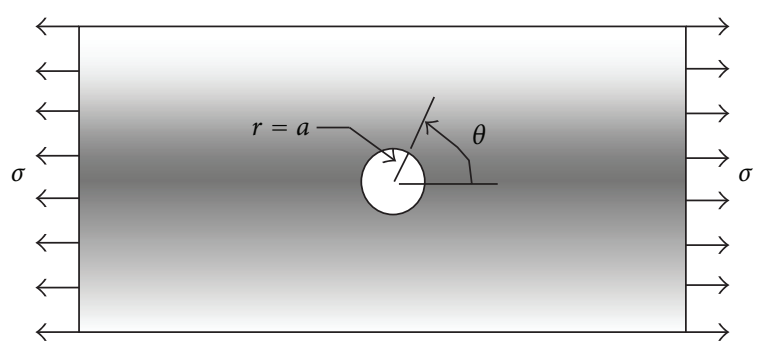

Figure 1: Plate model with center-hole.

form solution of the Von-Mises stress field in the plate with center hole.

\section{Theory of Analytical Model}

One can derive an expression representing the stress field in the plate shown in Figure 1 from Airy stress function [6]. The stress expression derived would be in polar coordinate where " $\sigma_{r}$ " (1) represents the stress in radial direction to the center hole. Likewise, " $\sigma_{\theta}$ " (2) represents the stress in the angular direction at circumference of the center hole. The " $\tau_{r \theta}$ " (3) would represent the shearing stress in polar coordinates. In all three expressions, " $r$ " represents the radial location, " $a$ " represents the center-hole radius, the " $\sigma$ " represents the normal stress due to normal loading, and " $\theta$ " represents the angular location from horizontal as shown by Figure 1:

$$
\begin{gathered}
\sigma_{r}=\frac{\sigma}{2}\left[\left(1-\frac{a^{2}}{r^{2}}\right)+\left(1+\frac{3 a^{4}}{r^{4}}-\frac{4 a^{2}}{r^{2}}\right) \cos 2 \theta\right] \\
\sigma_{\theta}=\frac{\sigma}{2}\left[\left(1+\frac{a^{2}}{r^{2}}\right)-\left(1+\frac{3 a^{4}}{r^{4}}\right) \cos 2 \theta\right] \\
\tau_{r \theta}=-\frac{\sigma}{2}\left(1-\frac{3 a^{4}}{r^{4}}+\frac{2 a^{2}}{r^{2}}\right) \sin 2 \theta .
\end{gathered}
$$

Further, the stress in polar coordinates can be expressed in terms of the stresses in Cartesian coordinate systems as shown by (4), (5), and (6). The " $\sigma_{x}$ " represents the normal stress, the " $\sigma_{y}$ " represents the transverse stress, and " $\tau_{x y}$ " represents the shear stress in Cartesian coordinate system:

$$
\begin{gathered}
\sigma_{r}=\sigma_{x} \cos ^{2} \theta+\sigma_{y} \sin ^{2} \theta+2 \tau_{x y} \sin \theta \cos \theta, \\
\sigma_{\theta}=\sigma_{x} \sin ^{2} \theta+\sigma_{y} \cos ^{2} \theta-2 \tau_{x y} \sin \theta \cos \theta, \\
\tau_{r \theta}=\left(\sigma_{y}-\sigma_{x}\right) \sin \theta \cos \theta+\tau_{x y}\left(\cos ^{2} \theta-\sin ^{2} \theta\right) .
\end{gathered}
$$

The expressions in (4), (5), and (6) can be formulated in matrix and vector forms, and the simultaneous equations can be solved for the stresses in the Cartesian coordinate. From the Cartesian stress expressions, the principal stress values can be determined by the following expression:

$$
\sigma_{1,2}=\frac{\sigma_{x}+\sigma_{y}}{2} \pm \sqrt{\left(\frac{\sigma_{x}-\sigma_{y}}{2}\right)^{2}+\tau_{x y}^{2}} .
$$

Finally, one can derive the Von-Mises stress $\left(\sigma_{\mathrm{von}}\right)$ representing the stress field on the plate by expressions in (8) where, $\sigma_{1}, \sigma_{2}$, and $\sigma_{3}$ are the principal stresses determined by (7). This Von-Mises stress would be used to draw the theoretical closed-form stress solution of the plate with a central hole:

$$
\sigma_{\text {von-mises }}=\sqrt{\frac{\left(\sigma_{1}-\sigma_{2}\right)^{2}+\left(\sigma_{2}-\sigma_{3}\right)^{2}+\left(\sigma_{1}-\sigma_{3}\right)^{2}}{2}} .
$$

\section{Finite Element Analysis Comparison Approach}

A quarter symmetric model of a thin plate with center hole is built with 4-node shell elements in ANSYS. Four distinct meshes are generated to elaborate the shape dependency of the FEA results. The first model is built with free-mesh of shell elements that are of fairly equal sides (LD free mesh). Figure 2(a) illustrates this unstructured mesh. The same model is built from a high-density free mesh (HD map mesh). In this mesh, in the immediate proximity of the hole area, where there is stress concentration, the mesh density is higher. Figure 2(b) illustrates this final mesh. A third model is built with mapped mesh of shell elements that are of almost equal sizes but have high aspect ratios (HAR map mesh). That is, the ratio of the long side of the element to the shorter side is large (slender shape). Also, these elements follow a uniform pattern. Figure 3(a) illustrates the shape and pattern of this structured mesh. The fourth model is built with mapped mesh of shell elements that have a low aspect ratio. That is, the ratio of the longer side of the elements to the shorter side is small (LAR map mesh). Figure 3(b) clearly illustrates the shape and pattern of this structured mesh. These four mesh models adequately summarize all of the possible mesh patterns that could be generated to mesh this plate geometry.

Symmetric properties are used in order to model only one-fourth of the full plate model. Symmetric boundary conditions are used at the left and bottom sides of the plate models. For applied loading, a $50 \mathrm{lb} /$ in uniform line pressure is applied to the right side of the model (depicted by red arrows in the meshed figure). This $50 \mathrm{lb}$ /in line loading is equivalent to a quasi-static $1000 \mathrm{lbf}$ tensile load applied to the plate thickness, in the right side. The top side of the plate model is free from restraints.

Table 1 is used to index the four different mesh-type models with respect to the number of elements, element size, and aspect ratio.

The "HD mesh" is the high-density free mesh, "LD free mesh" is the low density free mesh, "LAR map mesh" is the low aspect ratio mapped mesh, and "HAR map mesh" is the high aspect ratio mapped mesh. This comparison table materializes the mesh represented in Figures 2 and 3.

\section{Analytical Results}

Figures 4, 5, 6, and 7 represent the whole-field displacements for the free mesh, high aspect ratio mapped mesh, low aspect ratio mapped mesh, and the high-density mesh, respectively. All four models produce identical displacement counters of the plate model with the same maximum displacements. 
TABLE 1: The mesh-type index for all models.

\begin{tabular}{lcccc}
\hline Mesh type & HD mesh & LD free mesh & LAR map mesh & HAR map mesh \\
\hline Number of plate elements & 482 & 395 & 60 & 640 \\
Average size $\left(\right.$ inch $^{2}$ ) & 0.08 & 0.25 & 3 & 0.5 \\
Percentage of plate elements with high aspect ratio & 0 & 0 & 40 & 100 \\
Percentage of plate elements with low aspect ratio & 100 & 100 & 60 & 0 \\
\hline
\end{tabular}

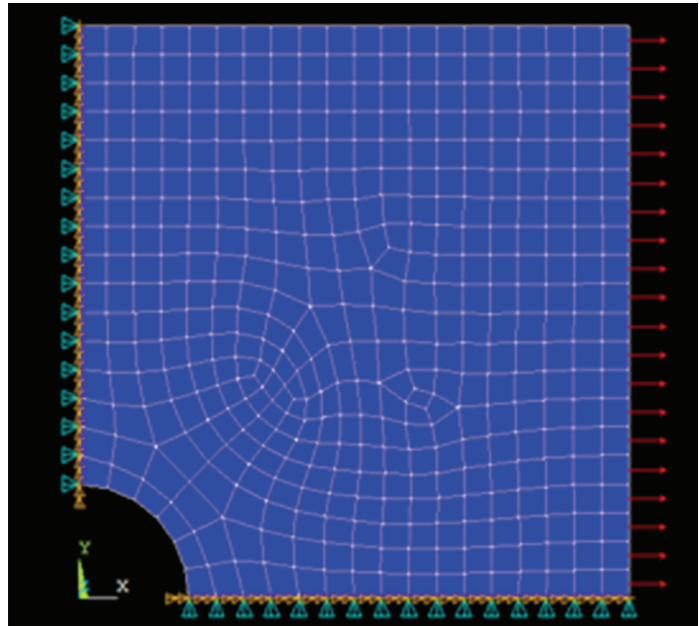

(a)

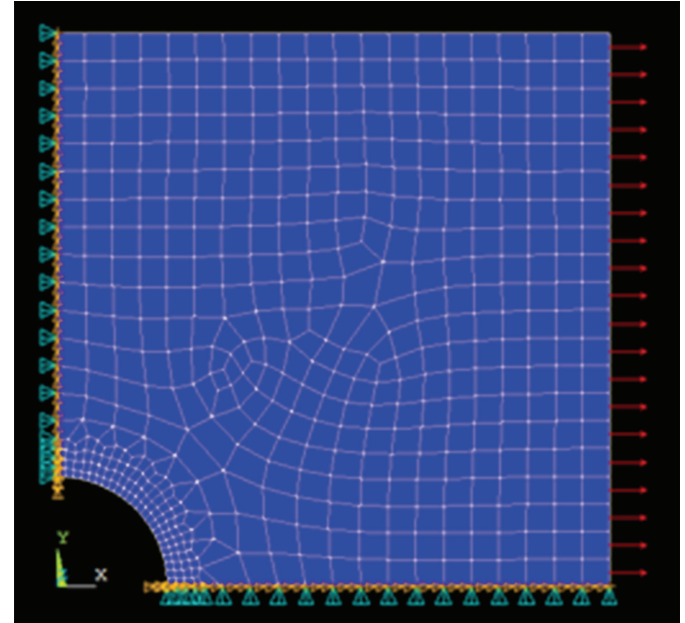

(b)

Figure 2: (a) The free mesh and (b) high-density free mesh using membrane shell elements.

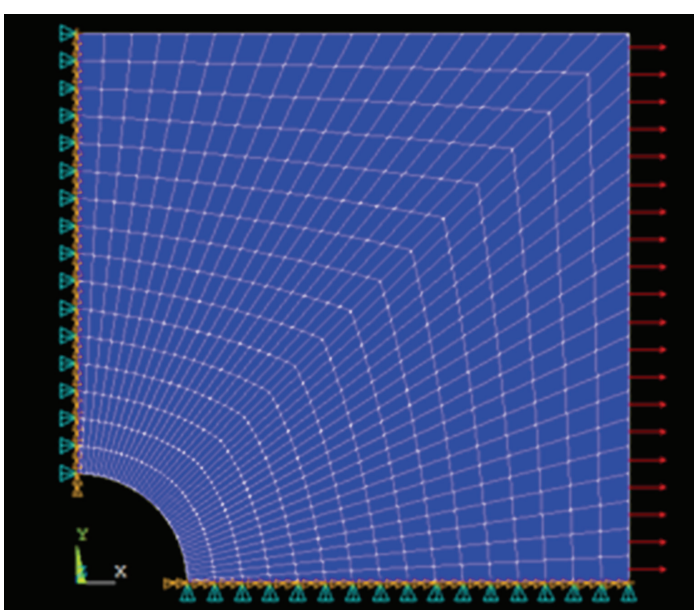

(a)

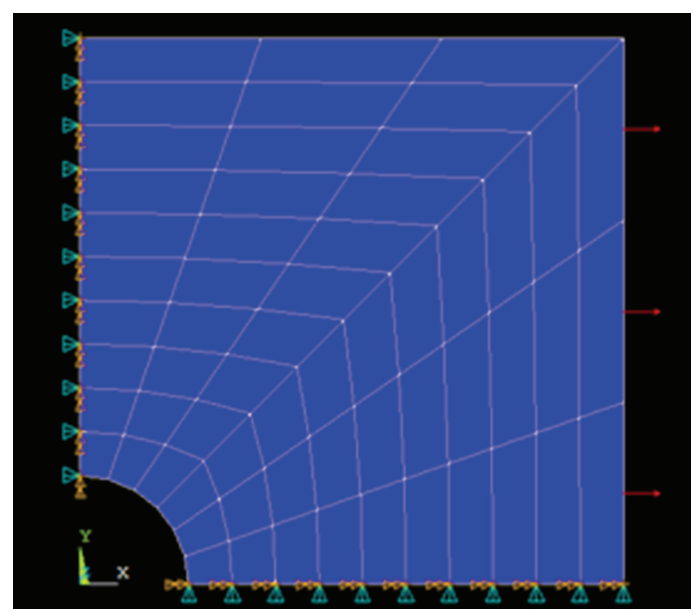

(b)

FIGURE 3: (a) The high aspect ratio mapped mesh and (b) low aspect ratio mapped mesh using membrane shell elements.

The displacement magnitudes indicate that the model is stiff and contour patterns indicate all four models are strained in the same manner.

The stress contours representing the stress field of the plate model are plotted in Figures 8, 9, 10, and 11. All of the plots, from the four different possible meshes, indicate a maximum Von-Mises magnitude at the 90 degree location around the hole at $r=2 \mathrm{inch}$. This is a stress concentration location which is also expected by theoretical values where displacement in the $x$-direction is considered to be constrained. Comparing all models, the stress contours over the entire plate model are similar in pattern. The only variations are the magnitudes of the stress levels developed in the plate stress field. The model with a denser mesh captures the stress levels more accurately as it will be shown by Figure 14 later. The models with mapped mesh produce a less accurate representation of the Von-Mises stresses.

The results from the finite element analysis of the previous four mesh models are compared with the theoretical Von-Mises stress levels derived by using the expression in (8). 


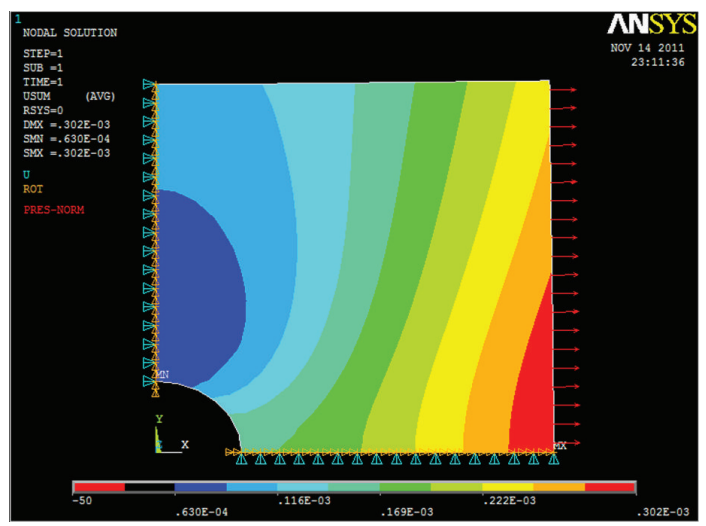

Figure 4: Displacement contour for the unstructured free-mesh model.

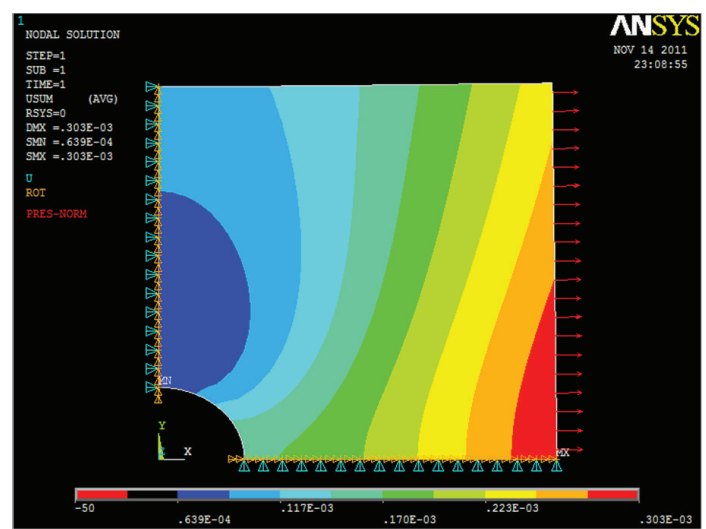

FIGURE 5: Displacement contour for the structured high aspect ratio mapped-mesh model.

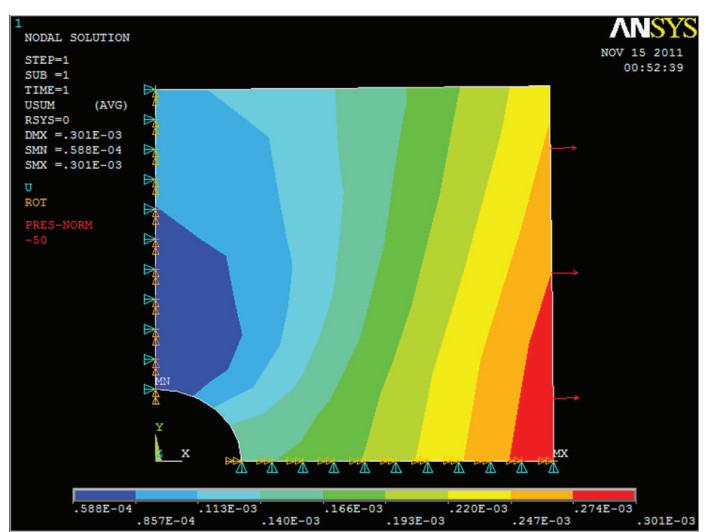

FIgURE 6: Displacement contour for the structured low aspect ratio mapped-mesh model.

Figure 12 draws a comparison between the Von-Mises stress-magnitude-results developed based on the different mesh generations. The Von-Mises stresses illustrated are of the stress contours immediately at the edge of the 2 inch radius hole section ( $r=2$ inch). The figure compares different mesh results with one another and to the results developed from closed-form stress analysis theory. A close

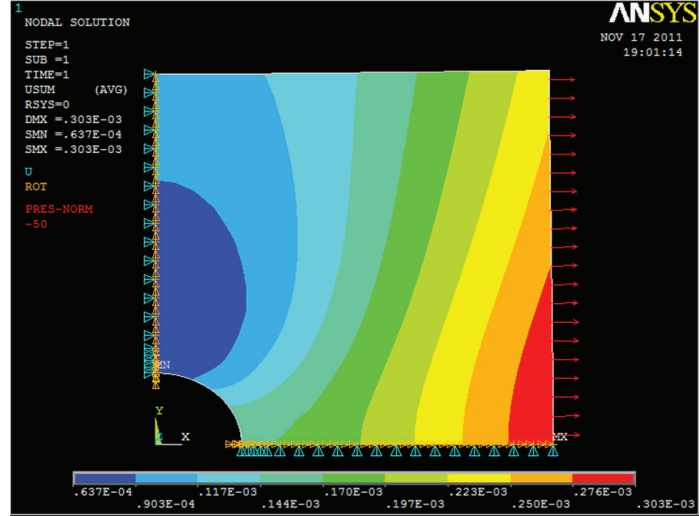

FIGURE 7: Displacement contour for the unstructured high-density free-mesh model.

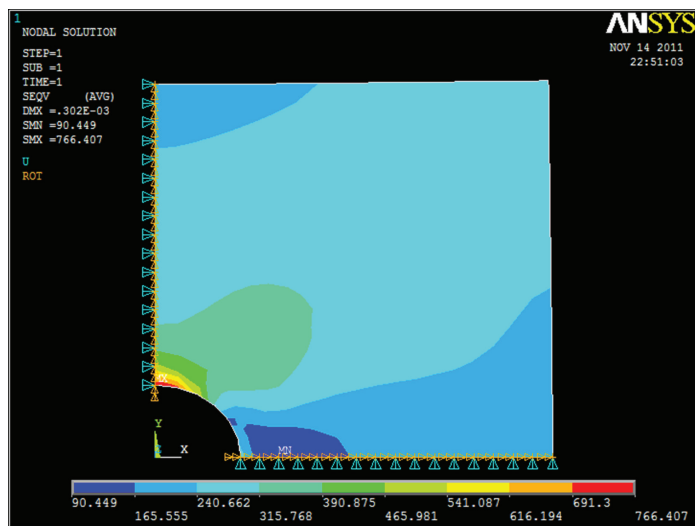

FIGURE 8: Stress contour for the unstructured free-mesh model.

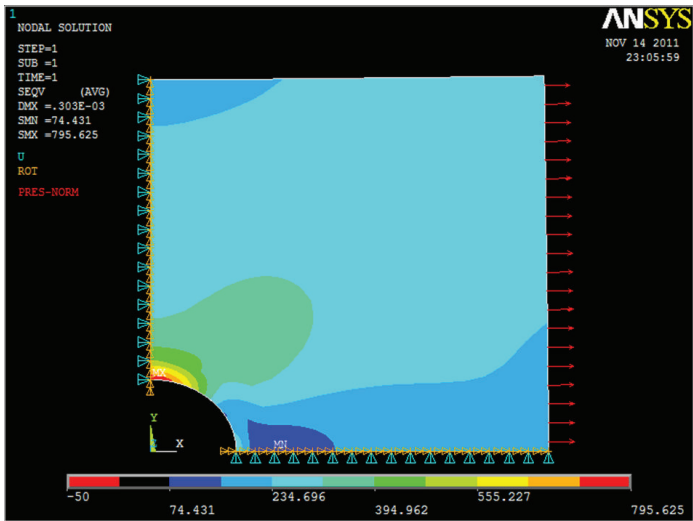

FIGURE 9: Stress contour for the structured high aspect ratio mapped-mesh model.

comparison of the different mesh models used, indicates that a "free-mesh" with fairly consistent elements matches the results of a high aspect ratio (HAR) "map-mesh." There exists an exception that there is a $3.65 \%$ difference in the final maximum Von-Mises stress due to the same loading for the two different meshes.

In comparison to the low aspect ratio (LAR) "mapmesh" results, the low aspect ratio mesh over estimates the 


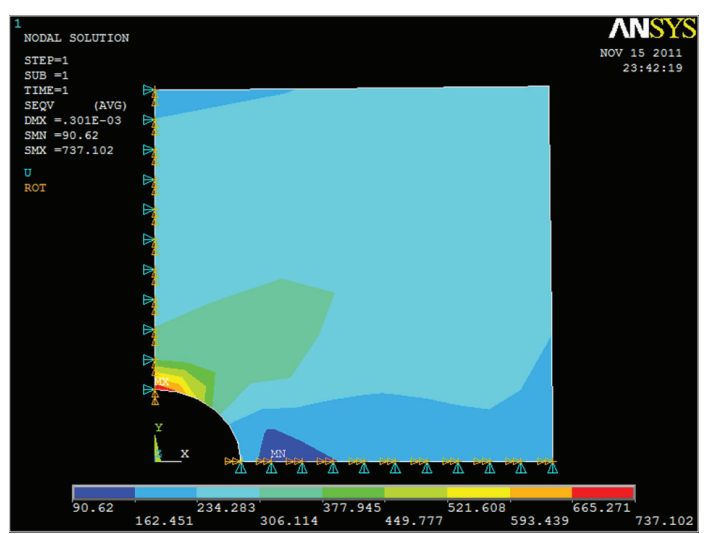

Figure 10: Stress contour for the structured low aspect ratio mapped-mesh model.

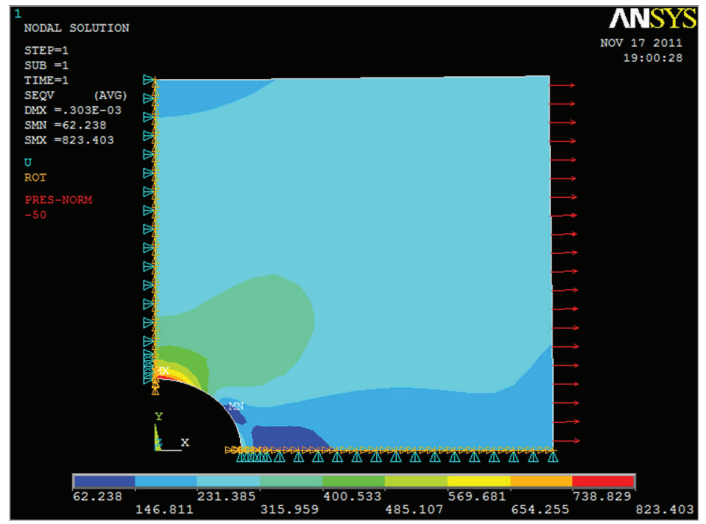

FIGURE 11: Stress contour for the unstructured high density freemesh model.

Von-Mises behavior of the plate slightly. However, for the low aspect ratio model, the final maximum Von-Mises stress magnitude is closer to the theoretical value with only a $1.72 \%$ difference. All three meshes together are close to the theoretical Von-Mises stress values except at 30 degrees angle location where the dip in the stress nullity is not very well determined. The high-density mesh (HD free mesh) closely follows the low-density free mesh and the high aspect ratio mapped mesh with the exception that it estimates the theoretical stress deep closer than the other three models. This mesh over estimates the theoretical final maximum stress level by $9.73 \%$. Yet it proves to be a sufficient mesh since it closely predicts the stress behavior as seen by Figure 12 .

Interestingly, from all of the four mesh models, it can be concluded that the proper aspect ratioed mapped mesh of the elements alone does not guaranty accurate results. An adequate mesh density is required to predict the stress behavior of a structure. Also, it could be concluded that low aspect ratio meshes are a good tool for determining margins of safety, since they may closely predict the maximum stress levels.

Most finite element analysis packages in some way provide meshing tools that generate well-behaved (moderate aspect ratio) elements. Yet none of them provide for an automatic mesh density measure. The analyst has to select

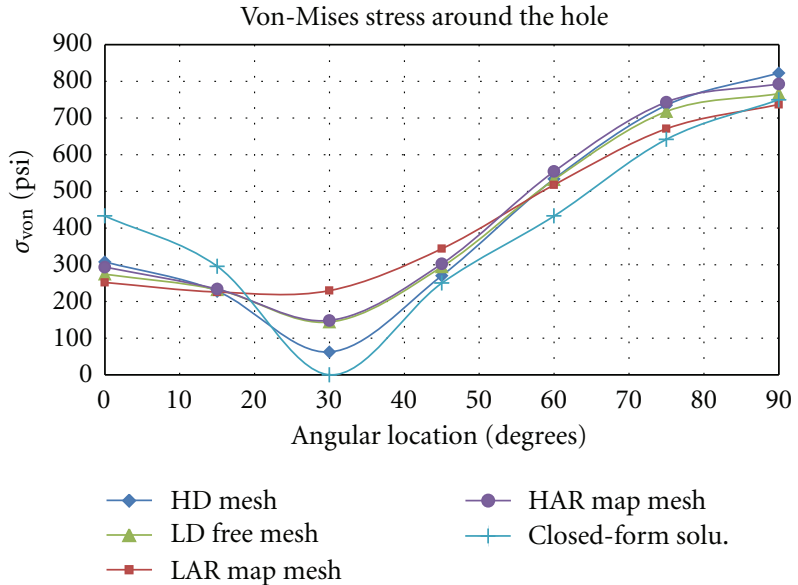

FIGURE 12: Von-Mises stress levels around the plate hole radius of different mesh configurations.

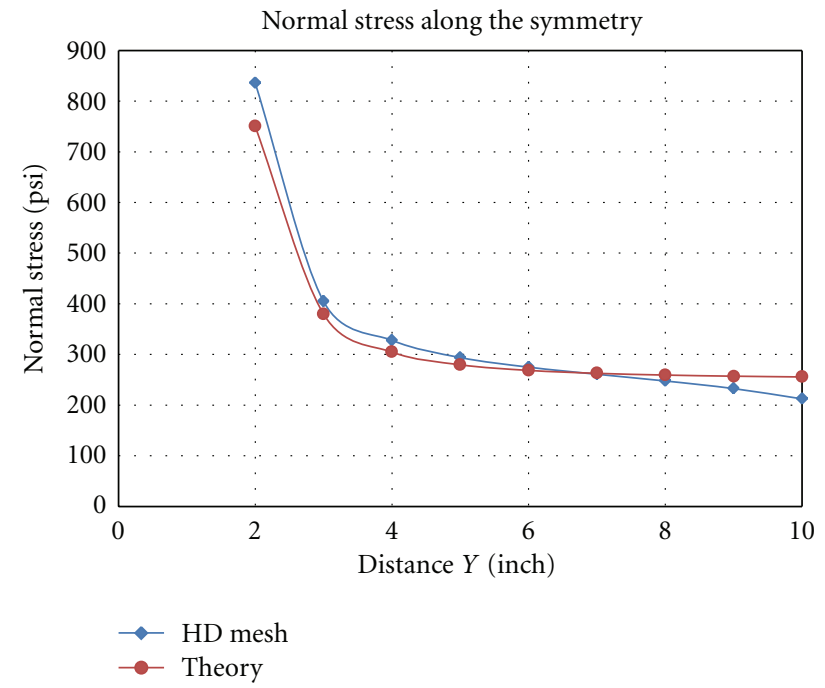

FIGURE 13: The normal stress versus the vertical distance to the top.

and control the mesh density of the region of the interest being analyzed. This has to be done either by preliminary FEA trial runs detecting the stress concentration regions or predetermination of the specified region by theoretical means. In either way, also the degree mesh density refinement has to be dealt with that only comes with FEA experience of similar structures.

To elaborate on the far-field accuracy of the most accurate mesh (the high-density mesh-HD mesh), the plot of the normal stress versus the vertical distance from the hole to the horizontal edge of the plate in $y$-direction (at the left symmetry line) is plotted in Figure 13. The FEA results from the high-density mesh minics the theoretical values of the normal stress on the plate accurately. It is conclusive that the high-density mesh also is a good far-field indicator.

\section{Finite Element Methods Theory}

To explain the theoretical reasoning for having less accuracy for high aspect ratio elements, it is essential to understand the 
computational method for the finite element modeling. One can begin by understanding the isoparametric formulation of elements [7]. For every element as illustrated by Figure 14, there is a linear displacement function defined in horizontal direction $u$ and vertical direction $v$ :

$$
\begin{aligned}
& u=\beta 1+\beta 2 x-\beta 3 y+\beta 4 x y \\
& v=\beta 5+\beta 6 x-\beta 7 y+\beta 8 x y .
\end{aligned}
$$

Using the boundary conditions at each node, the $\beta$ 's can be eliminated and displacement functions can be expressed as

$$
\begin{array}{r}
u=\left(\frac{1}{4 b h}\right)[(b-x)(h-y) u 1+(b+x)(h-y) u 2 \\
+(b+x)(h+y) u 3+(b-x)(h+y) u 4] \\
v=\left(\frac{1}{4 b h}\right)[(b-x)(h-y) v 1+(b+x)(h-y) v 2 \\
+(b+x)(h+y) v 3+(b-x)(h+y) v 4]
\end{array}
$$

The displacement expressions in (10) can be expressed in matrix form as

$$
\{U\}=[N]\{d\}
$$

where $[N]$ is the shape function and $\{d\}$ is the nodal displacement vector.

The relation (11) can be expanded and

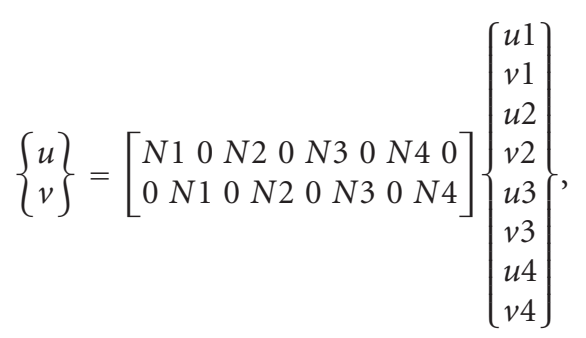

where $N i$ coefficients are the shape functions in terms of $x, y, b$, and $h$.

Now the strain in terms of the displacement is

$$
\left\{\begin{array}{c}
\varepsilon x \\
\varepsilon y \\
\gamma x y
\end{array}\right\}=\left\{\begin{array}{c}
\frac{\partial u}{\partial x} \\
\frac{\partial v}{\partial y} \\
\frac{\partial u}{\partial y}+\frac{\partial v}{\partial x}
\end{array}\right\}
$$

Which is

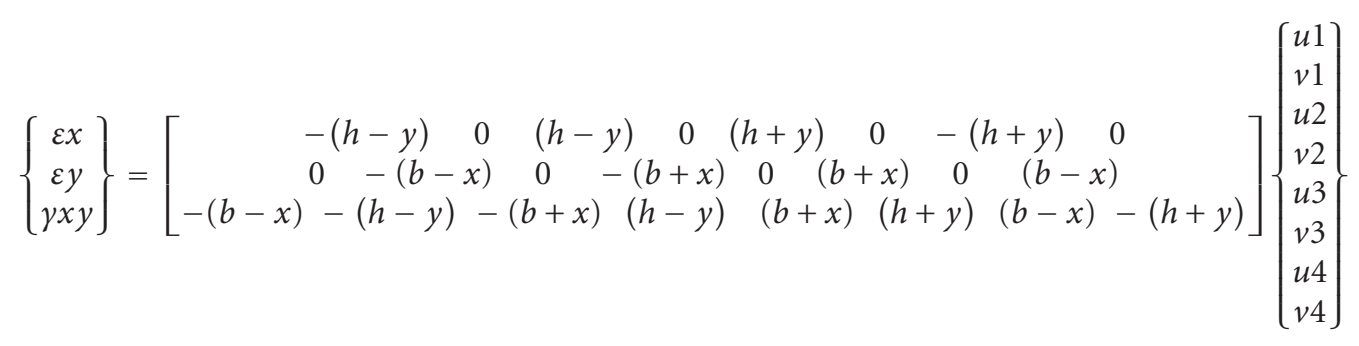

whereas for a plane stress condition, the stress vector $\{\sigma\}$ in terms of the element strains can be derived as

$$
\{\sigma\}=[D]\{\varepsilon\}
$$

where $D$ is the material stiffness matrix

$$
D:=\frac{E}{1-v^{2}} \cdot\left[\begin{array}{ccc}
1-v & v & 0 \\
v & 1 & 0 \\
0 & 0 & \frac{(1-v)}{2}
\end{array}\right] .
$$

Now from expression (15), a comparison between the stress results from a high aspect ratio element and a low aspect ratio element can be computed and one would have a higher stress value for the high aspect ratio element.

\section{Conclusions}

Four different mesh types consisting of a combination of structured and unstructured mesh where generated in the plate with central hole. High aspect ratio and low aspect ratio elements were generated for these models. The VonMises stresses and the plate displacements were developed and compared to each other. The results were in comparison to theoretical solutions. It was concluded that the proper aspect ratioed mapped mesh of the elements alone does not guarantee accurate results. An adequate mesh density is required to predict the stress behavior of a structure. A higher mesh density in the stress concentration region produces a high performance finite element analysis. The correct elemental aspect ratio helps the solution convergence but the mesh density increases the actual accuracy of the analysis. Further work will be carried on in concentration on the elemental formulation in FEA and its effects on the solution accuracy of the structured and unstructured meshing and produced in another paper. The subject is a universal subject for all available FEA packages since they use similar algorithms. 


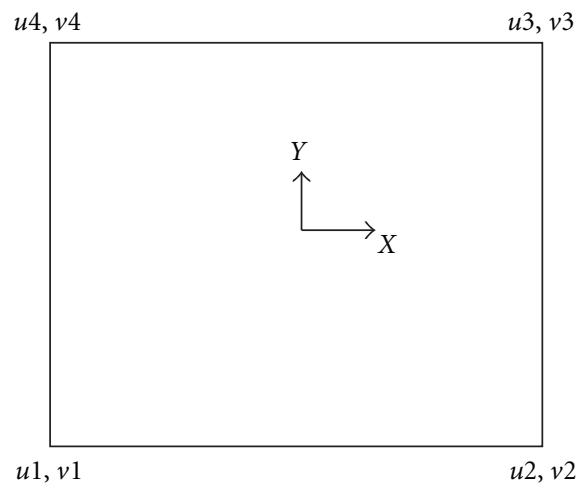

FIgURE 14: Four-node rectangular element.

\section{References}

[1] E. Madenci and I. Guven, The Finite Element Method and Applications in Engineering Using ANSYS, Springer, 2006.

[2] Phase 2, "Mesh generation tools", 2011, http://www.rockscience .com/.

[3] M. Picasso, "Adaptive finite elements with large aspect ratio based on an anisotropic error estimator involving first order derivatives," Computer Methods in Applied Mechanics and Engineering, vol. 196, no. 1-3, pp. 14-23, 2006.

[4] G. Asmar and E. Chakar, "Analysis of an isotropic plate containing three identical circular holes arranged in a triangular configuration," in Proceedings of the International Conference on Advances in Computational Tools for Engineering Applications (ACTEA '09), pp. 62-67, July 2009.

[5] K. M. El-Sawy and M. I. Martini, "Stability of biaxially loaded square plates with single central holes," Ships and Offshore Structures, vol. 5, no. 4, pp. 283-293, 2010.

[6] A. Javidinejad, Essentials of Mechanical Stress Analysis, 2008.

[7] D. L. Logan, Finite Element Method, Wadsworth Group, 2002. 

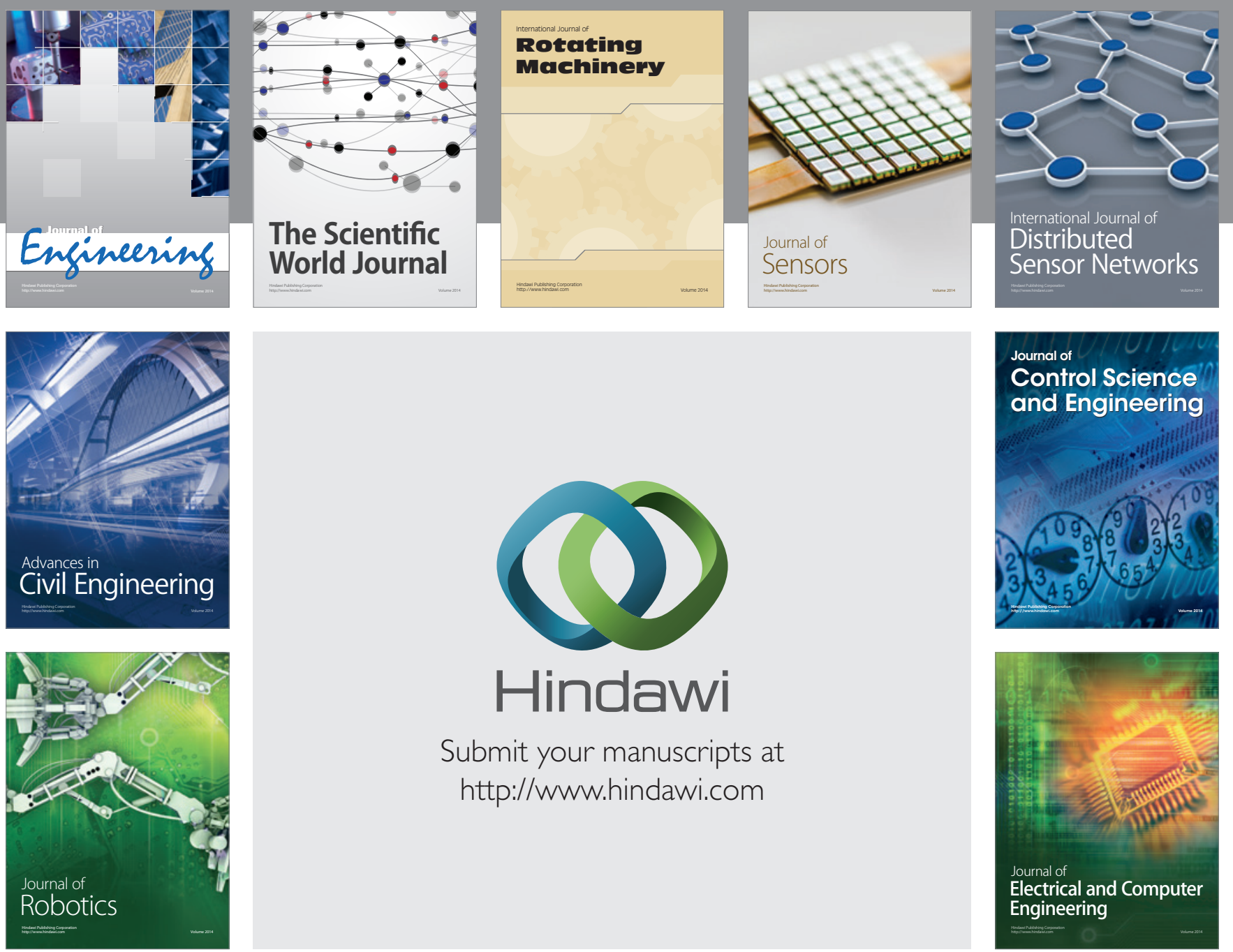

Submit your manuscripts at

http://www.hindawi.com
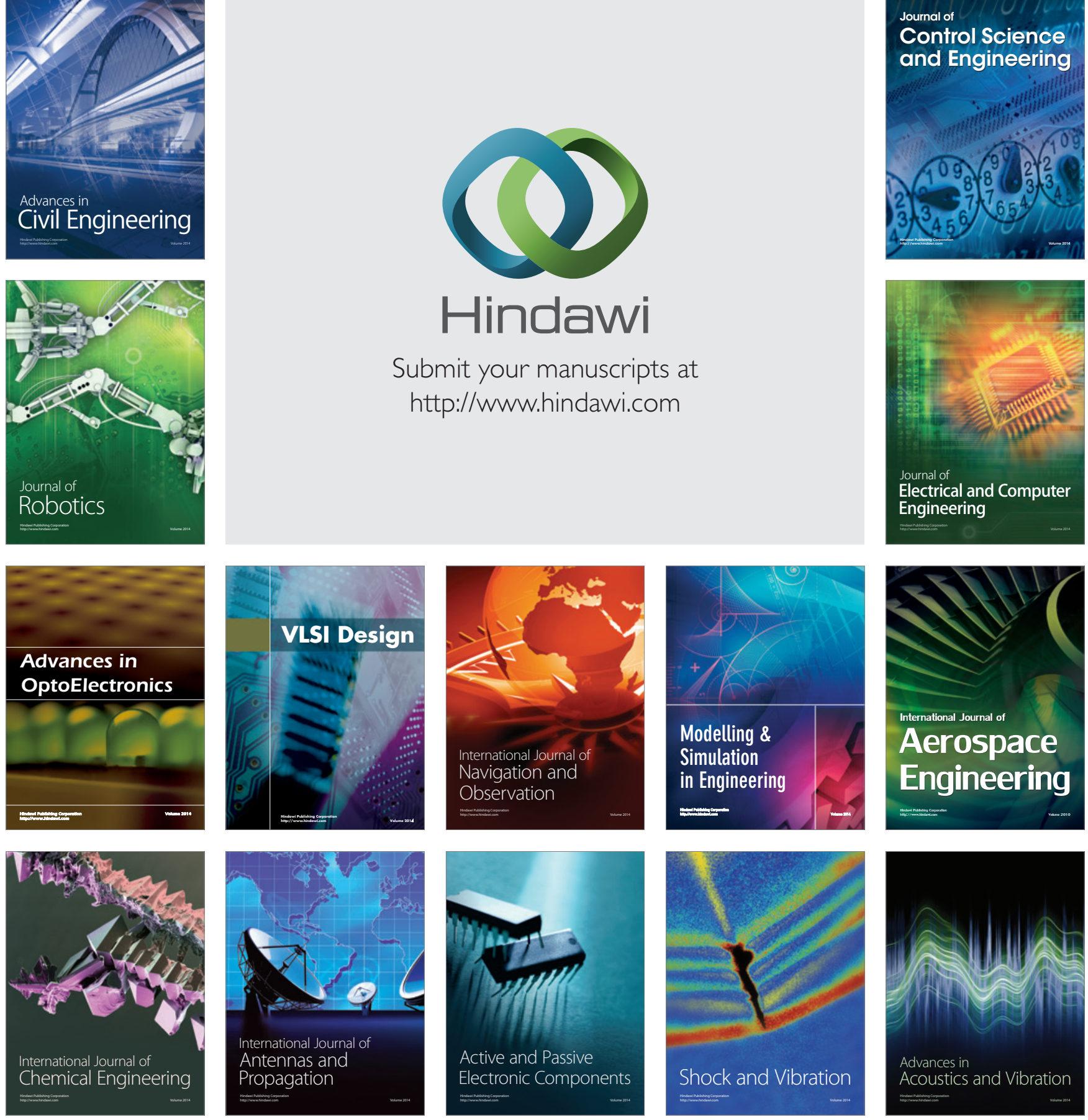